Journal of Applied AnALysis

Vol. 9, No. 2 (2003), pp. 187-200

\title{
A PRIORI SOLUTION ESTIMATES FOR NONLINEAR VECTOR INTEGRAL EQUATIONS
}

\author{
M. I. GIL'
}

Received April 23, 2002 and, in revised form, December 10, 2002

\begin{abstract}
Nonlinear vector integral equations are considered. Solution estimates and solvability conditions are derived. Applications to the periodic boundary value problem are also discussed. Under some restrictions our results improve the well-known ones. The main tool in the paper is the recent estimates for the resolvent of Hilbert-Schmidt operators.
\end{abstract}

\section{Introduction and statement of the main result}

A lot of papers and books are devoted to nonlinear integral equations, cf. [2], [9], [14], [15]. In this paper we suggest a new approach to deriving a priori solution estimates and solvability conditions for nonlinear vector integral equations. That approach is based on the recent estimates for the resolvent of linear Hilbert-Schmidt operators. As it is shown in Section 6 below, in appropriate situations our results improve the well known ones. In particular, we establish estimates for the amplitude of periodic solutions.

2000 Mathematics Subject Classification. 45G15, 45M15.

Key words and phrases. Nonlinear vector integral equations, solution estimates, solvability, periodic boundary value problem.

This research was supported by the Kamea Fund.

ISSN 1425-6908 C Heldermann Verlag. 
Let $E$ be a compact subset of a real Euclidean space $\mathbb{R}^{m}$, and $\mathbb{C}^{n}$ a complex Euclidean space with the Euclidean norm $\|\cdot\|_{\mathbb{C}^{n}}$. In addition, $L^{2}=L^{2}\left(E, \mathbb{C}^{n}\right)$ is the space of functions defined on $E$ with values in $\mathbb{C}^{n}$ and equipped with the norm

$$
\|h\|_{L^{2}}=\left[\int_{E}\|h(x)\|_{\mathbb{C}^{n}}^{2} d x\right]^{1 / 2} \quad\left(h \in L^{2}\left(E, \mathbb{C}^{n}\right)\right),
$$

and $C\left(E, \mathbb{C}^{n}\right)$ is the space of continuous functions defined on $E$ with values in $\mathbb{C}^{n}$ and equipped with the sup-norm $\|\cdot\|_{C}$.

For a positive $r \leq \infty$, put

$$
\omega_{r}=\left\{z \in \mathbb{C}^{n}:\|z\|_{\mathbb{C}^{n}} \leq r\right\} \text { and } \Omega_{r}(C)=\left\{h \in C\left(E, \mathbb{C}^{n}\right):\|h\|_{C} \leq r\right\} .
$$

Consider the equation

$$
u(x)=\int_{E} K(x, s, u(s)) u(s) d s+f(x) \quad(x \in E)
$$

where $f \in C\left(E, \mathbb{C}^{n}\right)$ and $K$ is an $n \times n$-matrix defined on $E^{2} \times \omega_{r}$. In addition, $K(x, s, z)$ is continuous in $z \in \omega_{r}$ for almost all $s \in E$ and

$$
\lim _{x \rightarrow x_{0}} \int_{E} \sup _{z \in \omega_{r}}\left\|K\left(x_{0}, s, z\right)-K(x, s, z)\right\|_{\mathbb{C}^{n}} d s=0 \quad\left(x_{0} \in E\right) .
$$

Put

$$
Q_{r}(x, s):=\sup _{z \in \omega_{r}}|K(x, s, z)|
$$

for almost all $s \in E$. Here $|K(x, s, z)|$ means the matrix whose entries are the absolute values of the entries of $K(x, s, z)$. So $Q_{r}(x, s)$ is a nonnegative $n \times n$-matrix.

Assume that

$$
q\left(Q_{r}\right):=\sup _{x \in E}\left[\int_{E}\left\|Q_{r}(x, s)\right\|_{\mathbb{C}^{n}}^{2} d s\right]^{1 / 2}<\infty .
$$

Let $B$ be an $m$-dimensional Hilbert-Schmidt operator $(m \leq \infty)$. The following quantity plays a key role in this paper:

$$
g(B):=\left(N^{2}(B)-\sum_{k=1}^{m}\left|\lambda_{k}(B)\right|^{2}\right)^{1 / 2},
$$

where $\lambda_{k}(B), k=1,2, \ldots, m$ are the eigenvalues of $B$ taking with their multiplicities and $N(B)$ is its Hilbert-Schmidt norm, i.e.

$$
N^{2}(B)=\text { Trace }\left(B B^{*}\right) \text {. }
$$

The asterisk means the adjointness. The following relations are true: $g^{2}(B) \leq$ $N^{2}(B)-\mid$ Trace $B^{2} \mid$ and

$$
g^{2}(B) \leq \frac{1}{2} N^{2}\left(B^{*}-B\right),
$$


cf. [5, Corollary 3.4.6]. Furthermore, introduce in $L^{2}$ the operator $\tilde{Q}_{r}$ by

$$
\left(\tilde{Q}_{r} v\right)(x)=\int_{E} Q_{r}(x, s) v(s) d s \quad\left(v \in L^{2}\left(E, \mathbb{C}^{n}\right)\right) .
$$

Due to (1.3), $\tilde{Q}_{r}$ is a Hilbert-Schmidt operator. Assume that the spectral radius $r_{s}\left(\tilde{Q}_{r}\right)$ of $\tilde{Q}_{r}$ is less than one:

$$
\rho_{s}\left(\tilde{Q}_{r}\right)<1
$$

and put

$$
\Theta\left(Q_{r}\right)=\sum_{k=0}^{\infty} \frac{g^{k}(\tilde{Q})}{\sqrt{k !}\left(1-\rho_{s}\left(\tilde{Q}_{r}\right)\right)^{k+1}} .
$$

Now we are in a position to formulate the main result of the paper

Theorem 1.1. Let the conditions (1.3), (1.5) and

$$
q\left(Q_{r}\right) \Theta\left(Q_{r}\right)\|f\|_{L^{2}}+\|f\|_{C}<r
$$

hold. Then equation (1.1) has at least one solution $u \in \Omega_{r}(C)$, satisfying the estimates

$$
\|u\|_{L^{2}} \leq \Theta\left(Q_{r}\right)\|f\|_{L^{2}}
$$

and

$$
\|u\|_{C} \leq q\left(Q_{r}\right) \Theta\left(Q_{r}\right)\|f\|_{L^{2}}+\|f\|_{C} \leq r .
$$

The proof of this theorem is divided into a series of lemmas, which are presented in the next section.

Note that due to (1.4)

$$
g^{2}\left(\tilde{Q}_{r}\right) \leq \frac{1}{2} N^{2}\left(\tilde{Q}_{r}^{*}-\tilde{Q}_{r}\right)=\frac{1}{2} \int_{E} \int_{E} \sum_{j, k=1}^{n}\left|b_{j k}(x, s)-b_{j k}(s, x)\right|^{2} d x d s
$$

where $b_{j k}$ are the entries of $Q_{r}(x, s)$. If $Q_{r}$ is a symmetric kernel, i.e. $b_{j k}(x, s)=b_{j k}(s, x)$, then $g\left(\tilde{Q}_{r}\right)=0$. Now Theorem 1.1 implies

Corollary 1.2. Let conditions (1.3) and (1.5) hold. If, in addition, $Q_{r}$ is a symmetric kernel, and

$$
\frac{q\left(Q_{r}\right)\|f\|_{L^{2}}}{1-\rho_{s}\left(\tilde{Q}_{r}\right)}+\|f\|_{C}<r
$$

then equation (1.1) has at least one solution $u \in \Omega_{r}(C)$, satisfying the estimates

and

$$
\|u\|_{L^{2}} \leq \frac{\|f\|_{L^{2}}}{1-\rho_{s}\left(\tilde{Q}_{r}\right)}
$$

$$
\|u\|_{C} \leq \frac{q\left(Q_{r}\right)\|f\|_{L^{2}}}{1-\rho_{s}\left(\tilde{Q}_{r}\right)}+\|f\|_{C}
$$


By virtue of Schwarz's inequality, it is not hard to check that

$$
\Theta\left(Q_{r}\right) \leq \frac{\sqrt{2}}{1-\rho_{s}\left(\tilde{Q}_{r}\right)} \exp \left[\frac{g^{2}(\tilde{Q})}{\left(1-\rho_{s}\left(\tilde{Q}_{r}\right)\right)^{2}}\right]
$$

\section{Proof of Theorem 1.1}

Let $X$ be a Banach space with a norm $\|\cdot\|_{X}$ and the unit operator $I$. In addition, $Y \subseteq X$ is a continuously imbedded Banach subspace with a norm $\|\cdot\|_{Y}$. For a positive number $r \leq \infty$, put $\Omega_{r}(Y) \equiv\left\{h \in Y:\|h\|_{Y} \leq r\right\}$. Consider the equation

$$
u=A(u) u+f
$$

where $A(h)$, for all $h \in \Omega_{r}(Y)$, is a linear operator acting from $X$ into $Y$ and $f \in Y$ is given. Moreover, there is a constant $q(Y, X)$, such that

$$
\|A(h) w\|_{Y} \leq q(Y, X)\|w\|_{X} \quad(w \in X ; h \in \Omega(Y)) .
$$

We will say that the mapping $\Phi: \Omega_{r}(Y) \rightarrow Y$ defined by

$$
\Phi(h) \equiv A(h) h+f\left(h \in \Omega_{r}(Y)\right)
$$

has the fixed point property in $Y$ if the uniform bound $\left\|v_{t}\right\|_{Y} \leq r$ for all possible solutions $v_{t}$ of the equation

$$
v=t A(v) v+t f \quad(0 \leq t \leq 1)
$$

implies the solvability of equation (2.1).

For instance, $\Phi$ can be a continuous compact mapping [12], [15] or a continuous condensing one (the Schaefer principle) [13], [1, Section 3.9.2], [4].

Lemma 2.1. Let $\Phi$ have the fixed-point property in $Y$. In addition, let the conditions (2.2) and

$$
J_{A}:=\sup _{h \in \Omega_{r}(Y), t \in[0,1]}\left\|(I-t A(h))^{-1}\right\|_{X}<\infty
$$

hold. Then equation (2.1) has at least one solution $u \in \Omega_{r}(Y)$ satisfying the estimates

$$
\|u\|_{X} \leq J_{A}\|f\|_{X}
$$

and

$$
\|u\|_{Y} \leq q(Y, X) J_{A}\|f\|_{X}+\|f\|_{Y}
$$

provided

$$
q(Y, X) J_{A}\|f\|_{X}+\|f\|_{Y}<r
$$


Proof. First, let $r=\infty$. Equation (2.3) is equivalent to the equation

$$
v=t(I-t A(v))^{-1} f .
$$

Hence, a possible solution $v_{t}$ of $(2.3)$ satisfies the inequalities

$$
\left\|v_{t}\right\|_{X} \leq t\left\|\left(I-t A\left(v_{t}\right)\right)^{-1} f\right\|_{X} \leq J_{A}\|f\|_{X} \quad(0 \leq t \leq 1) .
$$

This proves inequality (2.5). Moreover, from (2.3) it follows that

$$
\begin{aligned}
\left\|v_{t}\right\|_{Y} & \leq\left\|A\left(v_{t}\right) v_{t}\right\|_{Y}+\|f\|_{Y} \leq q(Y, X)\left\|v_{t}\right\|_{X}+\|f\|_{Y} \\
& \leq q(Y, X) J_{A}\|f\|_{X}+\|f\|_{Y} \leq q(Y, X) J_{A}\|f\|_{X}+\|f\|_{Y} .
\end{aligned}
$$

Since $\Phi$ has the fixed point property, equation (2.1) has a solution.

Let now $r<\infty$. Put $r_{1} \equiv q(Y, X) J_{A}\|f\|_{X}+\|f\|_{Y}$. According to (2.7), $r_{1}<r$.

By the Urysohn theorem [3, p. 15], there is a continuous real function $\psi_{r}$ defined on $Y$, such that $\psi_{r}(h)=1\left(\|h\|_{Y} \leq r_{1}\right)$ and $\psi_{r}(h)=0\left(\|h\|_{Y} \geq r\right)$. Besides, $0 \leq \psi_{r}(h) \leq 1\left(\|h\|_{Y} \leq r\right)$. Thus

$$
\sup _{h \in Y, t \in[0,1]}\left\|\left(I-t \psi_{r}(h) A(h)\right)^{-1}\right\|_{X} \leq J_{A} .
$$

Put $\Phi_{r}(h)=\psi_{r}(h) A(h)+f$ and consider the equation

$$
v_{t}=t \Phi_{r}\left(v_{t}\right) \text {. }
$$

According to (2.7) and (2.8), a solution $v_{t}$ of equation (2.9) satisfies the uniform estimate $\left\|v_{t}\right\|_{Y} \leq r_{1}$. Since $\Phi_{r}$ and $\Phi$ coincide on $\Omega_{r_{1}}(Y)$ and $\Phi$ has the fixed point property, $\Phi_{r}$ also has the fixed point property. So equation (2.9) with $t=1$ has a solution $v_{1}$, satisfying estimates (2.5) and (2.6). But $\Phi_{r}\left(v_{1}\right)=\Phi(v)$. Thus, $v_{1}$ satisfies (2.1). This finishes the proof.

Now, let $X=H$ be a Hilbert lattice, cf. [11]. Assume that

$$
|A(h) w| \leq B|w| \quad\left(w \in H, h \in \Omega_{r}(Y)\right),
$$

where $B$ is a positive Hilbert-Schmidt operator and $|\cdot|$ means the absolute value [11]. We will use the following result: let $B$ be an $m$-dimensional Hilbert-Schmidt operator $(m \leq \infty)$. Then for any regular $\lambda \in \mathbb{C}$,

$$
\left\|(B-\lambda I)^{-1}\right\|_{H} \leq \sum_{k=0}^{m-1} \frac{g^{k}(B)}{\sqrt{k !} \rho^{k+1}(B, \lambda)}
$$

where $\rho(B, \lambda)$ is the distance between the spectrum $\sigma(B)$ of $B$ and a complex point $\lambda$ (see [5, Corollary 1.2.4 and Theorem 2.4.1]). Assume that the spectral radius $\rho_{s}(B)$ of $B$ is less than one:

$$
\rho_{s}(B)<1 \text {. }
$$

Then (2.10) implies

$$
\left\|(I-t A(h))^{-1}\right\|_{H} \leq\left\|(I-B)^{-1}\right\|_{H} \quad\left(h \in \Omega_{r}(Y), t \in[0,1]\right) .
$$


But due to (2.11) and (2.12),

$$
\left\|(B-I)^{-1}\right\|_{H} \leq \theta(B)
$$

where

$$
\theta(B):=\sum_{k=0}^{\infty} \frac{g^{k}(B)}{\sqrt{k !}\left(1-\rho_{s}(B)\right)^{k+1}} .
$$

So $J_{A} \leq \theta(B)$. Now the previous lemma yields

Lemma 2.2. Let $\Phi$ have the fixed-pont property in $Y \subseteq H$. In addition, let the conditions (2.2) with $X=H,(2.10),(2.12)$ and

$$
\|f\|_{H} q(Y, H) \theta(B)+\|f\|_{Y}<r
$$

hold. Then equation (2.1) has at least one solution $u \in \Omega_{r}(Y)$, satisfying the estimates

and

$$
\|u\|_{H} \leq \theta(B)\|f\|_{H}
$$

$$
\|u\|_{Y} \leq\|f\|_{H} q(Y, H) \theta(B)+\|f\|_{Y} .
$$

Proof of Theorem 1.1. Due to the well-known Theorem X.1.2 [14], the nonlinear integral operator defined by the right-hand part of equation (1.1) is compact as operator acting from $C$ to $C$. Now the required result directly follows from the previous lemma with $Y=C\left(E, \mathbb{C}^{n}\right), H=L^{2}\left(E, \mathbb{C}^{n}\right)$ and $B=\tilde{Q}$.

\section{Equations "close" to Volterra ones}

In this section we are going to specialize Theorem 1.1 in the case of equations which are "close" to Volterra ones.

Let $x=\left(x_{k}\right), s=\left(s_{k}\right) \in E$. Under condition (1.2), put

$$
Q_{r}^{+}(x, s)=Q_{r}(x, s) \text { if } x_{k}<s_{k} \text { and } Q_{r}^{+}(x, s)=0 \text { for other } x, s .
$$

Define on $L^{2}$ an operator $V_{r}$ by

$$
\left(V_{r} v\right)(x)=\int_{E} Q_{r}^{+}(x, s) v(s) d s \quad\left(x \in E, v \in L^{2}\right)
$$

and put $T_{r}=\tilde{Q}_{r}-V_{r}$. Since under condition (1.3), $Q_{r}$ is a Hilbert-Schmidt kernel, the Volterra operator $V_{r}$ is a Hilbert-Schmidt one:

$$
N^{2}\left(V_{r}\right)=\int_{E} \int_{E}\left\|Q_{r}^{+}(x, s)\right\|_{\mathbb{C}^{n}}^{2} d s d x<\infty
$$

Clearly,

$$
\left\|T_{r}\right\|_{L^{2}}^{2} \leq \int_{E} \int_{E}\left\|Q_{r}(x, s)-Q_{r}^{+}(x, s)\right\|_{\mathbb{C}^{n}}^{2} d x d s
$$


Due to Theorem 3.5 from [6], the spectral radius $\rho_{s}\left(\tilde{Q}_{r}\right)$ of $\tilde{Q}_{r}$ satisfies the inequality $\rho_{s}\left(\tilde{Q}_{r}\right) \leq \beta_{r}$, where $\beta_{r}$ is the extreme right (positive) root of the scalar equation

$$
\frac{2\left\|T_{r}\right\|_{L^{2}}^{2}}{y^{2}} \exp \left[\frac{2 N^{2}\left(V_{r}\right)}{y^{2}}\right]=1
$$

Let $\beta_{r}<1$. Then $\Theta\left(\tilde{Q}_{r}\right) \leq \theta\left(V_{r}, T_{r}\right)$, where

$$
\theta\left(V_{r}, T_{r}\right):=\frac{\sqrt{2}}{1-\beta_{r}} \exp \left[\frac{N^{2}\left(V_{r}\right)}{\left(1-\beta_{r}\right)^{2}}\right] .
$$

Now Theorem 1.1 implies

Theorem 3.1. Let the conditions (1.3), $\beta_{r}<1$ and

$$
q\left(Q_{r}\right) \theta\left(V_{r}, T_{r}\right)\|f\|_{L^{2}}+\|f\|_{C}<r
$$

hold. Then equation (1.1) has at least one solution $u \in \Omega_{r}(C)$, satisfying the estimates

$$
\|u\|_{L^{2}} \leq \theta\left(V_{r}, T_{r}\right)\|f\|_{L^{2}}
$$

and

$$
\|u\|_{C} \leq q\left(Q_{r}\right) \theta\left(V_{r}, T\right)\|f\|_{L^{2}}+\|f\|_{C} .
$$

Substituting $y^{2}=2 N^{2}\left(V_{r}\right) x$ in (3.1) we have

$$
\frac{\left\|T_{r}\right\|_{L^{2}}^{2}}{N^{2}\left(V_{r}\right) x} \exp \left[\frac{1}{x}\right]=1
$$

Now Lemma 4.6 from [7] yields the inequality $\beta_{r} \leq \delta_{r}$, where

$$
\delta_{r}:=\frac{\sqrt{2} N\left(V_{r}\right)}{\ln ^{1 / 2}\left[1 / 2+\sqrt{1 / 4+\left\|T_{r}\right\|_{L^{2}}^{-2} N^{2}\left(V_{r}\right)}\right]} .
$$

Thus the previous theorem implies

Corollary 3.2. Let the conditions (1.3) and $\delta_{r}<1$ hold. In addition, with the notation

let

$$
\theta\left(\delta_{r}, V_{r}\right):=\frac{\sqrt{2}}{1-\delta_{r}} \exp \left[\frac{N^{2}\left(V_{r}\right)}{\left(1-\delta_{r}\right)^{2}}\right]
$$

$$
q\left(Q_{r}\right) \theta\left(\delta_{r}, V_{r}\right)\|f\|_{L^{2}}+\|f\|_{C}<r
$$

Then equation (1.1) has at least one solution $u \in \Omega_{r}(C)$, satisfying the estimates

and

$$
\|u\|_{L^{2}} \leq \theta\left(\delta_{r}, V_{r}\right)\|f\|_{L^{2}}
$$

$$
\|u\|_{C} \leq q\left(Q_{r}\right) \theta\left(\delta_{r}, V_{r}\right)\|f\|_{L^{2}}+\|f\|_{C}
$$


Furthermore let $x=\left(x_{k}\right), s=\left(s_{k}\right) \in E$, again. Put

$$
Q_{r}^{-}(x, s)=Q_{r}(x, s) \text { if } x_{k}>s_{k} \text { and } Q_{r}^{-}(x, s)=0 \text { for other } x, s,
$$

and introduce the Volterra operator $\tilde{V}_{r}$ by

$$
\left(\tilde{V}_{r} v\right)(x)=\int_{\omega} Q_{r}^{-}(x, s) v(s) d s
$$

Then in our above arguments we can replace $V_{r}$ by operator $\tilde{V}_{r}$.

\section{Equations with convolution majorants}

Let $E=[-\pi, \pi]$ and conditions (1.2) and (1.3) hold with $Q_{r}(x, s)=$ $Q_{r}(x-s)$ where $Q_{r}(x)$ is a nonnegative $n \times n$ matrix defined on $\mathbb{R}$. That is,

$$
|K(x, s, z)| \leq Q_{r}(x-s) \quad\left(-\pi \leq x, s \leq \pi ; z \in \omega_{r}\right) .
$$

and

$$
q\left(Q_{r}\right)=\sup _{x \in[-\pi, \pi]}\left[\int_{-\pi}^{\pi}\left\|Q_{r}(x-s)\right\|_{\mathbb{C}^{n}}^{2} d s\right]^{1 / 2}<\infty .
$$

Therefore

$$
\left(\tilde{Q}_{r} v\right)(x)=\int_{-\pi}^{\pi} Q_{r}(x-s) v(s) d s \quad\left(v \in L^{2}\left([-\pi, \pi], \mathbb{C}^{n}\right)\right) .
$$

In this section we are going to improve Theorem 1.1 in the case (4.1). Let

$$
Q_{r}(x)=\sum_{k=-\infty}^{\infty} B_{k} e^{i k x}
$$

be the Fourier expansion of $Q_{r}$ with the matrix Fourier coefficients

$$
B_{k}=\frac{1}{2 \pi} \int_{-\pi}^{\pi} Q_{r}(s) e^{-i k s} d s
$$

Due to Lemma 4.1 from [6],

$$
\rho_{s}(\tilde{Q})=\sup _{l=0, \pm 1, \ldots} \rho_{s}\left(B_{l}\right) .
$$

where $\rho_{s}\left(B_{l}\right)$ is the spectral radius of matrix $B_{l}$. It is easy to check that under consideration,

$$
\left\|\left(\tilde{Q}_{r}-I\right)^{-1}\right\|_{L^{2}}=\sup _{l=0, \pm 1, \ldots}\left\|\left(B_{l}-I_{n}\right)^{-1}\right\|_{\mathbb{C}^{n}} .
$$

Here $I$ and $I_{n}$ are the unit operators in $L^{2}$ and $\mathbb{C}^{n}$, respectively. Assume that

$$
\sup _{l=0, \pm 1, \ldots} \rho_{s}\left(B_{l}\right)<1
$$


Then according to $(2.11)$

$$
\left\|\left(\tilde{Q}_{r}-I\right)^{-1}\right\|_{L^{2}} \leq \theta_{0}\left(Q_{r}\right)
$$

where

$$
\theta_{0}\left(Q_{r}\right):=\sup _{l=0, \pm 1, \ldots} \sum_{k=0}^{n-1} \frac{g^{k}\left(B_{l}\right)}{\sqrt{k !}\left(1-\rho_{s}\left(B_{l}\right)\right)^{k+1}} .
$$

Now Lemma 2.1 implies

Theorem 4.1. Let the conditions (4.1), (4.2), (4.4) and

$$
q\left(Q_{r}\right) \theta_{0}\left(Q_{r}\right)\|f\|_{L^{2}}+\|f\|_{C}<r
$$

hold. Then equation (1.1) with $E=[-\pi, \pi]$ has at least one solution $u \in \Omega_{r}(C)$, satisfying the estimates

$$
\|u\|_{L^{2}} \leq \theta_{0}\left(Q_{r}\right)\|f\|_{L^{2}}
$$

and

$$
\|u\|_{C} \leq q\left(Q_{r}\right) \theta_{0}\left(Q_{r}\right)\|f\|_{L^{2}}+\|f\|_{C}
$$

Let $Q_{r}(x)$ be a Hermitian matrix for all $x \in[-\pi, \pi]$. Then $B_{l}$ are normal matrices and $g\left(B_{l}\right)=0$. Thus Corollary 1.2 is true with $\rho_{s}\left(\tilde{Q}_{r}\right)$ defined by (4.3).

\section{The periodic boundary value problem}

Consider in $\mathbb{C}^{n}$ the problem

$$
\begin{aligned}
& d u / d x=W(x, u(x)) u(x)+f_{0}(x) \\
& u(-\pi)=u(\pi)
\end{aligned}
$$

where $f_{0} \in L^{2}\left([-\pi, \pi], \mathbb{C}^{n}\right)$ and $W(x, z)$ is an $n \times n$-matrix continuously dependent on $x \in[-\pi, \pi]$ and $z \in \omega_{r}$. The operator $S$ defined on $L^{2}$ by

$$
(S v)(x)=d u / d x+u
$$

with conditions (5.2) has the Green matrix-function $G(x, s)=G(x-s)$ defined by

$$
G(x-s)=\sum_{k=-\infty}^{\infty}(1+k i)^{-1} e^{i k(x-s)} I_{n} \quad(x, s \in[-\pi, \pi])
$$

where $I_{n}$ is the unit matrix. Hence $(5.1)$ can be written as

$$
u(x)=\int_{-\pi}^{\pi} G(x-s)\left(W(s, u(s))-I_{n}\right) u(s) d s+f(x)
$$


where

$$
f(x)=\int_{-\pi}^{\pi} G(x-s) f_{0}(s) d s .
$$

Since $G$ is a solution of the equation

$$
G^{\prime}+G=\delta(x)
$$

where $\delta(x)$ is the delta-Dirack function, we have

$$
G(x)= \begin{cases}C_{\pi} e^{-x} & \text { if } x<0 \\ \left(C_{\pi}+I_{n}\right) e^{-x} & \text { if } x \geq 0\end{cases}
$$

where

$$
C_{\pi}:=\frac{e^{-\pi}}{e^{\pi}-e^{-\pi}} I_{n}
$$

That is $G$ is positive. Let the condition

$$
\left|W(x, z)-I_{n}\right| \leq M_{r} \quad\left(z \in \omega_{r}\right)
$$

hold where $M_{r}$ is a nonnegative constant matrix. Then (4.1) is valid with $K(x, s, z)=G(x-s)\left(W(s, z)-I_{n}\right)$ and $Q_{r}(x-s)=G(x-s) M_{r}$. So according to (5.3) in the considered case the Fourier coefficients to $Q_{r}$ are

$$
B_{k}=(1+k i)^{-1} M_{r} .
$$

Moreover, the eigenvalues of $B_{k}$ are $\lambda_{j}\left(B_{k}\right)=(1+k i)^{-1} \lambda_{j}\left(M_{r}\right)(j=$ $1, \ldots, n)$. Hence,

$$
\rho_{s}\left(B_{k}\right)=\frac{\rho_{s}\left(M_{r}\right)}{\left(1+k^{2}\right)^{1 / 2}} \leq \rho_{s}\left(M_{r}\right)
$$

and

$$
g\left(B_{k}\right)=\frac{g\left(M_{r}\right)}{\left(1+k^{2}\right)^{1 / 2}} \quad(k=0, \pm 1, \pm 2, \ldots) .
$$

Consequently,

$$
\theta_{0}\left(Q_{r}\right)=\sup _{l=0, \pm 1, \ldots} \sum_{k=0}^{n-1} \frac{g^{k}\left(B_{l}\right)}{\sqrt{k !}\left(1-\rho_{s}\left(B_{l}\right)\right)^{k+1}} \leq \psi\left(M_{r}\right)
$$

where

$$
\psi\left(M_{r}\right):=\sum_{k=0}^{n-1} \frac{g^{k}\left(M_{r}\right)}{\sqrt{k !}\left(1-\rho_{s}\left(M_{r}\right)\right)^{k+1}} .
$$

Moreover, in the considered case, conditin (4.2) holds with

$$
q\left(Q_{r}\right) \leq\left\|M_{r}\right\|_{\mathbb{C}^{n}} \sup _{x}\left[\int_{-\pi}^{\pi} G^{2}(x-s) d s\right]^{1 / 2} .
$$

According to (5.3), simple calculations show that

$$
\sup _{x}\left[\int_{-\pi}^{\pi} G^{2}(x-s) d s\right]^{1 / 2} \leq \beta_{\pi}
$$


where

$$
\beta_{\pi}:=\left[2 \pi \sum_{k=-\infty}^{\infty} \frac{1}{1+k^{2}}\right]^{1 / 2} .
$$

So condition (4.2) holds with

$$
q\left(Q_{r}\right)=\beta_{\pi}\left\|M_{r}\right\|_{\mathbb{C}^{n}}
$$

Moreover, due to the Schwarz inequality

$$
\|f\|_{C}=\sup _{x}\left\|\int_{-\pi}^{\pi} G(x-s) f_{0}(s) d s\right\|_{\mathbb{C}^{n}} \leq \beta_{\pi}\left\|f_{0}\right\|_{L^{2}} .
$$

Hence,

$$
\|f\|_{L^{2}} \leq \sqrt{2 \pi}\|f\|_{C} \leq \sqrt{2 \pi} \beta_{\pi}\left\|f_{0}\right\|_{L^{2}}
$$

Now Theorem 4.1 and relations (5.7) and (5.8) yield

Theorem 5.1. Let the conditions (5.6) and $\rho_{s}\left(M_{r}\right)<1$ hold. In addition, let

$$
\left\|f_{0}\right\|_{L^{2}} \beta_{\pi}\left(\sqrt{2 \pi}\left\|M_{r}\right\|_{\mathbb{C}^{n}} \psi\left(M_{r}\right)+1\right)<r .
$$

Then problem (5.1), (5.2) has at least one continous solution $u \in \Omega_{r}(C)$, satisfying the estimates

$$
\|u\|_{L^{2}} \leq\left\|f_{0}\right\|_{L^{2}} \beta_{\pi} \sqrt{2 \pi} \psi\left(M_{r}\right)
$$

and

$$
\|u\|_{C} \leq\left\|f_{0}\right\|_{L^{2}} \beta_{\pi}\left(\sqrt{2 \pi}\left\|M_{r}\right\|_{\mathbb{C}^{n}} \psi\left(M_{r}\right)+1\right) .
$$

Note that if $M_{r}$ is a Hermitian matrix, then

$$
\psi\left(M_{r}\right) \leq \frac{1}{1-\rho_{s}\left(M_{r}\right)}
$$

In the general case, thanks to $(1.4), g^{2}\left(M_{r}\right) \leq N^{2}\left(M_{r}-M_{r}^{*}\right) / 2$.

\section{Concluding remarks}

In this section we are going to compare our results with the well-known ones.

1. Take $E=[0,1]$ and consider the Volterra equation

$$
u(x)-\int_{0}^{x} K(x, s, u(s)) u(s) d s=f(x) \quad(x \in[0,1])
$$

where $f \in C\left([0,1], \mathbb{C}^{n}\right)$ and $K$ is the same as in Section 1 with $E=[0,1]$. In addition, $Q_{r}(x, s)(0 \leq s \leq x \leq 1)$ defined by (1.2) has the property

$$
q\left(Q_{r}\right)=\sup _{0 \leq x \leq 1}\left[\int_{0}^{x}\left\|Q_{r}(x, s)\right\|_{\mathbb{C}^{n}}^{2} d s\right]^{1 / 2}<\infty
$$


Simple calculations show that in the considered case

$$
g^{2}\left(\tilde{Q}_{r}\right)=\int_{0}^{1} \int_{0}^{x}\left\|Q_{r}(x, s)\right\|_{\mathbb{C}^{n}}^{2} d s d x \leq q^{2}\left(Q_{r}\right)
$$

and $\rho_{s}\left(\tilde{Q}_{r}\right)=0$. Moreover,

$$
\Theta\left(Q_{r}\right) \leq \sum_{k=0}^{\infty} \frac{q^{k}\left(Q_{r}\right)}{\sqrt{k !}} \leq \sqrt{2} \exp \left[q^{2}\left(Q_{r}\right)\right] .
$$

Obviously, under consideration, $\|f\|_{L^{2}} \leq\|f\|_{C}$. Let the condition

$$
\|f\|_{C}\left(1+q\left(Q_{r}\right) \sqrt{2} \exp \left[q^{2}\left(Q_{r}\right)\right]\right)<r
$$

hold. Then due to Theorem 1.1 and inequality (1.9), equation (6.1) has at least one solution $u \in \Omega_{r}(C)$. Let us compare this result with the following well known solvability conditions for equation (1.1) in the scalar case [14, Chapter X, Theorem 2.4]:

$$
K \text { continuously maps }[0,1]^{2} \times \Omega_{r}(C) \text { into } \mathbb{C},
$$

and

$$
r \sup _{0 \leq x, s \leq 1 ;|z| \leq r}|K(x, s, z)|+\|f\|_{C}<r .
$$

Or, equivalently,

$$
r \sup _{0 \leq x, s \leq 1}\left|Q_{r}(x, s)\right|+\|f\|_{C}<r .
$$

About other solvability conditions see for instance [15, p. 59].

Theorem 1.1 does not require condition (6.3). In particular, $K$ can have integrable singularities with respect to the second argument. But even under condition (6.3), relation (6.2) with $n=1$ improves (6.4), since (6.5) requires the inequality

$$
\sup _{0 \leq x, s \leq 1 ;|z| \leq r}|K(x, s, z)|<1 .
$$

At the same time due to (6.2), $K$ can be "arbitrary" if $f$ is sufficiently small. So (6.2) improves (6.4) for Volterra equations of the type (6.1) and equations which are "close" to (6.1).

The same reasonings are valid for the equation

$$
u(x)-\int_{x}^{1} K(x, s, u(s)) u(s) d s=f(x) \quad(0 \leq x \leq 1)
$$

and equations which are "close" to it.

2. Now let us consider the Anselone-Moor system from elasticity theory $[2$, Section 1.2]

$$
\begin{array}{r}
u_{j}(x)-\gamma \int_{0}^{1}\left(L_{j 1}(x, s) u_{1}(s) u_{2}(s)+L_{j 2}(x, s) u_{1}^{2}(s)\right) d s=f_{j}(x) \\
(x \in[0,1] ; \gamma=\text { const }>0)
\end{array}
$$


where $L_{j 2}(x, s)$ are bounded measurable kernels. The following solvability conditions for system (6.6) are well known [2, p. 10]:

$$
4 \gamma\|f\|_{C} \max _{j=1,2} \sup _{x, s \in[0,1]}\left(\left|L_{j 2}(x, s)\right|+\left|L_{j 2}(x, s)\right|\right)<1 .
$$

Let us improve this result in the case of the Volterra system

$$
\begin{array}{r}
u_{j}(x)-\gamma \int_{0}^{x}\left(L_{j 1}(x, s) u_{1}(s) u_{2}(s)+L_{j 2}(x, s) u_{1}^{2}(s)\right) d s=f_{j}(x) \\
(x \in[0,1]) .
\end{array}
$$

Put

$$
\begin{aligned}
& K_{j 1}(x, s, z)=\gamma L_{j 2}(x, s) z_{1}, K_{j 2}(x, s, z)=\gamma L_{j 1}(x, s) z_{1} \\
&\left(z=\left(z_{1}, z_{2}\right) \in \mathbb{C}^{2} ; j=1,2\right) .
\end{aligned}
$$

Then (6.8) takes the form (6.1). According to (1.2) put

$$
Q_{r}(x, s)=r \gamma|L(x, s)|
$$

where $|L(x, s)|$ is the $2 \times 2$-matrix whose entries are the absolute values of the matrix

$$
L(x, s)=\left(L_{j k}(x, s)\right)_{j, k=1}^{2} .
$$

Clearly, condition (1.3) holds with $q\left(Q_{r}\right)=\gamma r \nu(L)$, where

$$
\nu(L)=\left[\sup _{x} \int_{0}^{x}\|L(x, s)\|_{\mathbb{C}^{2}}^{2} d s\right]^{1 / 2} .
$$

Now (6.2) yields the following solvability conditions for system (6.8):

$$
\|f\|_{C}(1+\gamma r \nu(L) \sqrt{2} \exp [\gamma r \nu(L)])<r .
$$

Taking $r$ sufficiently small, due to Theorem 1.1, we can assert that system (6.8) is solvable for arbitrary $\gamma$, provided $\|f\|_{C}<r$. Thus, (6.9) improves (6.7) for the Volterra system and systems which are "close" to it.

3. Furthermore, let us consider periodic problem $(5.1),(5.2)$. Let $(\cdot, \cdot)$ denote the scalar product in $L^{2}\left([-\pi, \pi], \mathbb{C}^{n}\right)$. If one of the following conitions holds:

$$
(z, W(x, z)) \leq a(x)\left(\|z\|_{\mathbb{C}^{n}}^{2}+1\right) \quad\left(x \in[-\pi, \pi] ; z \in \mathbb{R}^{n}\right) .
$$

for a nonnegative function $a \in L^{1}[-\pi, \pi]$, or

$$
(z, W(x, z)) \leq 0 \quad\left(x \in[-\pi, \pi] ; z \in \omega_{r}\right),
$$

then the problem (5.1), (5.2) has a continous solution, cf. [10, p. 67, Corollaries VI.4 and VI.5 ] (see also [8] and [15]).

Clearly, our Theorem 5.1 allows us to consider more general nonlinearities than ones satisfying (6.10) and (6.11). In addition, Theorem 5.1 gives us estimates for the amplitude of oscillations. 


\section{References}

[1] Akhmerov, R. R., Kamenskiı̌, M. I., Potapov, S. A., Rodkina, A. E., Sadovskiǔ, B. N., Measures of Noncompactness and Condensig Operators (in Russian), "Nauka" Sibirsk, Novosibirsk, 1986.

[2] Argyros, I. Polynomial Operator Equations in Abstract Spaces and applications, CRC Press, Boca Raton, FL, 1998.

[3] Dunford, N., Schwartz, J. T., Linear Operators. Part I. General Theory, WileyInterscience Publishers, Inc., New York, Interscience Publishers, Ltd., London, 1958.

[4] Gatica, J. A., Fixed point theorems for $k$-set contractions and pseudo-contractive mappings, J. Math. Anal. Appl., 46(3) (1974), 555-564.

[5] Gil', M. I., Norm Estimations for Operator-Valued Functions and Applications, Marcel Dekker, Inc., New York, 1995.

[6] Gil', M. I., Invertibility conditions and bounds for spectra of matrix integral operators, Monatsh. Math. 129 (2000), 15-23.

[7] Gil', M. I., Invertibility and spectrum localization of non-selfadjoint operators, Adv. in Appl. Math., 28 (2002), 1-19.

[8] Krasnosel'skii, A. M., Asymptotics of Nonlinearities and Operator Equations, Birkhäuser Verlag, Basel, 1995.

[9] Krasnosel'skii, M. A., Zabreiko, P. P., Geometrical Methods in Nonlinear Analysis, Springer-Verlag, Berlin, 1984.

[10] Mawhin, J., Topological Degree Methods in Nonlinear Boundary Value Problems, Amer. Math. Soc., Providence, RI, 1979.

[11] Meyer-Nienberg, P., Banach Lattices, Springer-Verlag, Berlin, 1991.

[12] O'Regan, D., Precup, R., Theorems of Leray-Schauder Type and Applications, Series in Mathematical Analysis and Applications 3, Cordon and Breach Science Publishers, Amsterdam, 2001.

[13] Schaefer, H. Neue Existenzsätze in der Theorie nichtlinearer Integralgleichungen (in German), Ber. Verh. Sächs. Acad. Wiss. Leipzig. Math.-Nat. Kl. 101(7), 1955.

[14] Zabreiko, P. P., Koshelev, A. I., Krasnosel'skii, M. A., Mikhlin, S. G., Rakovshik, L. S., Stetzenko, B. Ya., Integral Equations, Noordhoff, Leiden, 1975.

[15] Zeidler, E., Nonlinear Functional Analysis and its Applications, Springer-Verlag, New York, 1986.

M. I. GIL'

Department of Mathematics

Ben Gurion University of the Negev

P.O. Box 653, BeER-Sheva 84105

ISRAEL

GILMI@CS.BGU.AC.IL 